\title{
F/H AREA ETF EFFLUENT (H-016 OUTFALL) CERIODAPHNIA SURVIVAL/REPRODUCTION TEST, TEST DATE: MARCH 21, 1991 (U)
}

by

W. L. Specht, et al.

Westinghouse Savannah River Company

Savannah River Laboratory

Aiken, South Carolina 29808

A Technical Report for Publication

\section{DISCLAIMER}

This report was prepared as an account of work sponsored by an agency of the United States Government. Neither the United States Government nor any agency thereof, nor any of their employees, makes any warranty, express or implied, or assumes any legal liability or responsibility for the accuracy, completeness, or usefulness of any information, apparatus, product, or process disclosed, or represents that its use would not infringe privately owned rights. Reference herein to any specific commercial product, process, or service by trade name, trademark, manufacturer, or otherwise does not necessarily constitute or imply its endorsement, recommendation, or favoring by the United Siates Government or any agency thereof. The views and opinions of authors expressed herein do not necessarily state or reflect those of the United States Government or any agency thereof.

This paper was prepared in connection with work done under Contract No. DE-AC09-89SR18035 with the U.S. Department of Energy. By acceptance of this paper, the publisher and/or recipient acknowledges the U.S. Government's right to retain a nonexclusive, royalty-free license in and to any copyright covering this paper, along with the right to reproduce and to authorize others to reproduce all or part of the copyrighted paper.

\section{MASTER}

BISTRIBUTION OF THIS OOCUMENT IS UNIIM!TEE
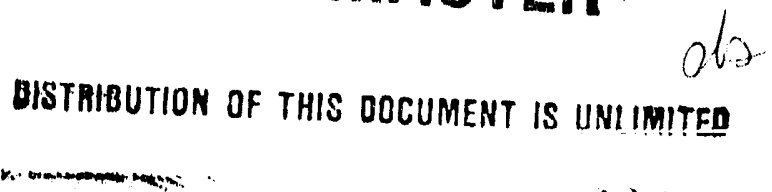
Derivative Classifier DBmou Luehow D. B. Moore-Shedrow, Section Manager Authorized Derivative Classifer

F/H AREA ETF EFFLUENT (H.016 OUTFALL) CERIODAPHNIA SURVIVAL/REPRODUCTION TEST, TEST DATE: MARCH 21, 1991 (U)

WSRC Technical Representative: W. L. Specht

Approved by :

D. B. Moore-Shedrow, Section Manager

Environmental Sciences Section

Savannah River Laboratory

Publication Date: August 1991

WESTINGHOUSE SAVANNAH RIVER COMPANY

SAVANNAH RIVER SITE

AIKEN, SC 29808

Prepared for the U.S. Department of Energy under Contract No. DE-AC09-88SR18035 
(1. Chem-Nuclear Laboratory Services, Inc.

Post Ollice Bux 17467

Greenville. South Carolina 29606

(803) 235.0707

\section{Ceriodaphnia SURVIVAL/RBPRODUCTION TEST}

$$
\text { Sample Identification: } \quad \text { H - F Area }
$$

Client: Savannah River Site

Location: Aiken, South Carolina

Test Date: March 21, 1991 


\section{IMPERPREMATION OF RBSULTS}

This toxicity test was conducted to determine if the effluent affects the survival or reproduction of the test organisms during a seven day period. The test involved exposing the test organisms to a series of dilutions of the effluent. At each dilution the survival and reproduction of ten test organisms was recorded. Each effluent dilution was compared to a control set of test organisms. Survival data were analyzed by Fisher's Exact Test and the Trimmed Spearman-Karber test to determine the effluent concentration necessary to cause statistically significant $(p=0.05)$ mortality. Reproduction data was analyzed for normality, homogeneity of variance and equality of replicates among dilutions to determine the appropriate statistical test for analysis of statistical differences in reproduction among dilutions. Results are summarized as follows:

Acute Toxicity:

Trimmed Spearman-Karber Analysis

LC50= 54.77 \& Effluent
$\begin{gathered}95 \% \text { Confidence Limits } \\ \text { Lower Upper }\end{gathered}$

Fisher's Exact Test

$\begin{array}{rr}\text { NOEC }= & 30.08 \text { Effluent } \\ \text { LOEC }= & 100.08 \text { Effluent } \\ \text { ChV }= & 54.768 \text { Effluent }\end{array}$

Chronic Toxicity

Bonferroni's t-test

(NOEC) No Observed Effect Conc.:

30.08

(LOEC) Lowest Observed Effect Conc.:

$100.0 \%$

chronic Value:

$54.76 \%$

$(p=0.05)$ 


\section{Client: Savannah River Site \\ Sample Identification: H - F Area \\ Start Date: March 21, 1991}

SURVIVAL DATA (AT Test Termination)

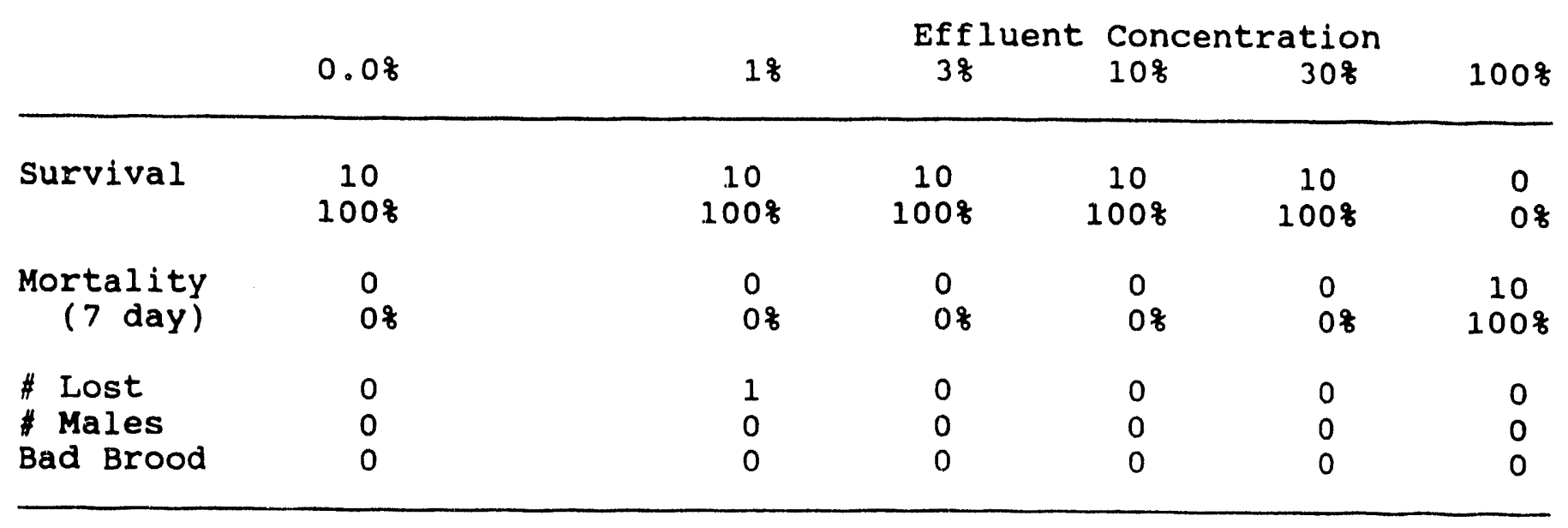

SURVIVAL - DATA ANALYSIS

Trimmed Spearman-Karber Analysis

(Probit Analysis cannot be used because there are not 2 effluent concentrations that have between 0 and 1008 mortality.)

LC50 $=54.77 \quad$ \& Effluent
Fisher's Exact Test
$\begin{array}{r}\text { NOEC }= \\ \text { LOEC }= \\ \text { ChV }=\end{array} \quad \begin{aligned} 100.08 \text { Effluent } \\ 54.768 \text { Effluent }\end{aligned}$

\section{Confidence Limits Lower Upper}

Not Reliable 


\section{RESOLTS}

Client: Savannah River site

Sample Identification: H - F Area

Start Date: March 21, 1991

CHRONIC TOXICITY - Daily Reproduction Totals

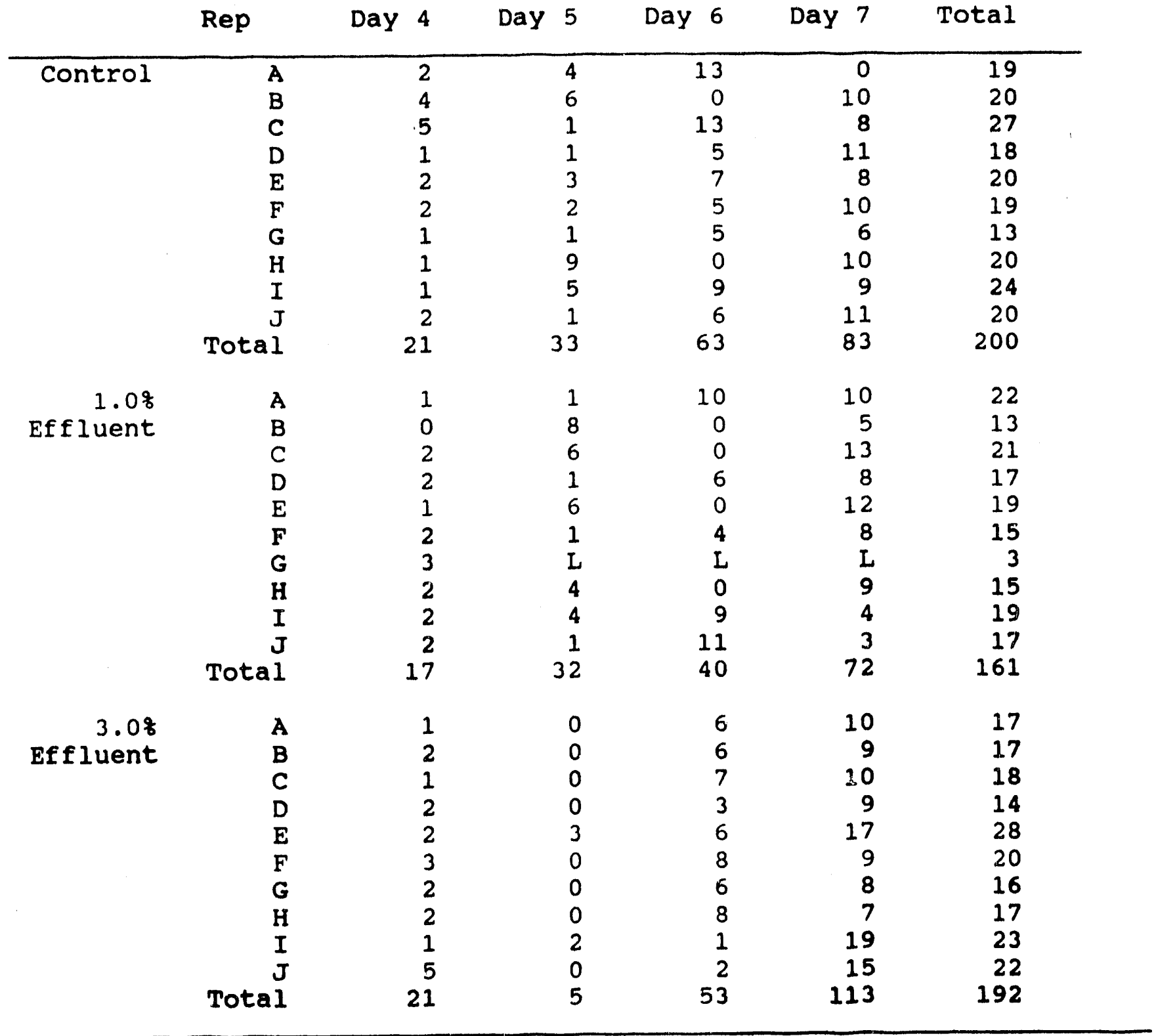




\section{Rasurus}

Client: Savannah River Site

Sample Identification: H - F Area

Start Date: March 21, 1991

CHRONIC TOXICITY - Daily Reproduction Totals

\begin{tabular}{ccrrrrrr} 
& Rep & Day 4 & Day 5 & Day 6 & Day 7 & Total \\
\hline Effluent & A & 3 & 7 & 12 & 0 & 22 \\
& B & 0 & 4 & 9 & 8 & 21 \\
& C & 3 & 1 & 6 & 9 & 19 \\
D & 1 & 0 & 4 & 17 & 22 \\
& E & 1 & 1 & 6 & 10 & 18 \\
F & 0 & 5 & 8 & 0 & 13 \\
G & 2 & 0 & 4 & 12 & 18 \\
H & 2 & 0 & 7 & 12 & 21 \\
& I & 2 & 0 & 6 & 13 & 21 \\
J & 2 & 4 & 0 & 17 & 23 \\
& Total & 16 & 22 & 62 & 98 & 198
\end{tabular}

30.08

Effluent

$\begin{array}{rrr}\text { A } & 2 & 0 \\ \text { B } & 0 & 1 \\ \text { C } & 1 & 6 \\ \text { D } & 1 & 5 \\ \text { E } & 2 & 0 \\ \text { F } & 0 & 4 \\ \text { G } & 2 & 6 \\ \text { H } & 0 & 10 \\ \text { I } & 2 & 2 \\ \text { J } & 1 & 0 \\ \text { Total } & 11 & 34\end{array}$

$100.0 \%$

Effluent

$\begin{array}{rrr}\text { A } & X & X \\ \text { B } & X & X \\ \text { C } & X & X \\ \text { D } & X & X \\ \text { E } & X & X \\ \text { F } & X & X \\ \text { G } & X & X \\ \text { H } & X & X \\ I & X & X \\ \text { J } & X & X \\ \text { Total } & 0 & 0\end{array}$

\begin{tabular}{|c|c|c|c|}
\hline$X$ & $X$ & $\mathbf{X}$ & 0 \\
\hline$x$ & $x$ & $x$ & 0 \\
\hline $\mathbf{x}$ & $x$ & $x$ & 0 \\
\hline$x$ & $X$ & $X$ & 0 \\
\hline$x$ & $x$ & X & 0 \\
\hline$x$ & $x$ & $X$ & 0 \\
\hline$x$ & $\mathrm{x}$ & $\mathrm{X}$ & 0 \\
\hline$x$ & $x$ & $x$ & 0 \\
\hline$x$ & $x$ & $\mathbf{X}$ & 0 \\
\hline $\mathrm{x}$ & $x$ & $\mathrm{X}$ & 0 \\
\hline 0 & 0 & 0 & 0 \\
\hline
\end{tabular}

$\mathrm{X}$ represents the mortality of the test organism. 


\section{R.SULTS}

Client: Savannah River site

Sample Identification: H - F Area

Start Date: March 21, 1991

CHRONIC TOXICITY - Statistical Analysis

ANOVA and Bonferroni's $t$ Test: (Assumes Data is Normally Distributed)

\begin{tabular}{|c|c|c|c|c|c|c|}
\hline conc. & 0.08 & 1.08 & 3.08 & 10.08 & 30.08 & 100.08 \\
\hline $\begin{array}{l}\text { Average Young } \\
\text { per Female }\end{array}$ & 20.0 & 17.6 & 19.2 & 19.8 & 15.6 & 0.0 \\
\hline Std. Dev. & 3.65 & 2.96 & 4.13 & 2.94 & 3.20 & 0 \\
\hline "t" value & & 1.557 & .523 & .131 & 2.879 & \\
\hline $\begin{array}{l}\text { Reproduction } \\
\text { significantly less } \\
\text { than control? } \\
\text { ( } 958 \text { confidence) }\end{array}$ & & no & no & no & no & yes \\
\hline
\end{tabular}

\section{$95 q$ conf.}

(NOEC) No Observed Effect Conc.: 30.08

(LOEC) Lowest Observed Effect Conc.: 100.0\%

Chronic Value:

54.768

The data are normally distributed.

The data are homogenous in variance.

The number of replicates in each concentration is not equal.

The appropriate statistical test is Bonferroni's t-test. 


\section{SANIPIMG INPORUATION}

Three effluent samples were collected by and transported to Chem-Nuclear iaboratory Services by on-site personnel. Samples were collected in $41 \mathrm{~L}$ plastic cubitainers.

\section{Client: Savannah River Site}

Sample Identification: H - F Area

Start Date: March 21, 1991

$$
\begin{aligned}
& \text { Sample Sample Sample } \\
& \text { \#1 \#2 \#3 }
\end{aligned}
$$

\section{Lab \#}

Type

Date sampled

Time sampled

Days of use

Maximum hold time:

Temperature Upon Receipt:
BI0811-1 BI0811-2 BI0811-3 grab grab grab

$3 / 20 / 913 / 22 / 91 \quad 3 / 25 / 91$ 11 AM $9: 30$ AM 12:11 PM
0,1
36 hrs
2,3
$4,5,6$
$4.0^{\circ} \mathrm{C}$
72 hrs
72 hrs
$0.5^{\circ} \mathrm{C}$
$1.0^{\circ} \mathrm{C}$

Effluent Field Parameters

D.O. (ppm)

$\mathrm{pH}$

Temp (C)

Res CI (ppm)

\section{Sample Sample Sample}

$\# 1$ \#2 \#3

8.2

$\begin{array}{lll}8.2 & \overline{7.23} & 7 \overline{7.61} \\ 18.46 \mathrm{C} & 18.0 \mathrm{C} & 22.0 \mathrm{C} \\ <0.05 & <0.05 & <0.05\end{array}$




\section{Client: Savannah River site}

\section{Start Date: March 21, 1991}

Test Type: 7 Day Chronic Toxicity - Reproduction Test

Test Location: Chem-Nuclear Laboratory Services

$\begin{array}{rll}\text { Test organism: Ceriedaphnia dubia } & \begin{array}{r}\text { Source: } \\ \text { Age: }\end{array} & 24 \mathrm{hrs}\end{array}$

Test Procedure: Short-Term Methods for Estimating the Chronic Toxicity of Effluents and Receiving Waters to Freshwater organisms. 2nd Edition. EPA 600/4-85/014 Method 1002

Test vessels: $\quad 30 \mathrm{ml}$ plastic beakers

Amount Test Soln: $15 \mathrm{ml} /$ replicate

\# Replicates/Concentration: 10 (test vessels randomized)

Test Organisms/Replicate: 1

\# of Test Concentrations Used (excluding control):

Test Solution Renewal: Daily

Food Source:

$0.10 \mathrm{ml}$ Selenastrum $\left(3.1-3.5 \times 10^{7} \mathrm{cells} / \mathrm{ml}\right)$ and $0.05 \mathrm{ml}$ YCT daily / test vessel

Test Perfomed BY:

Test Results and Report Prepared By:
Joel stephens

Analytical Biologist

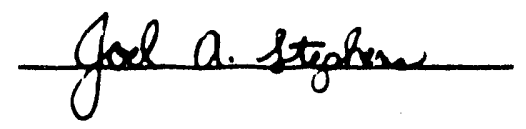

Joel stephens

Analytical Biologist

Goel a. steghem livisn 
Client: Savannah River site

Sample Identification: H - F Area

Start Date: March 21, 1991

Dilution water

ID: Upper Three Runs Creek Water

Preparation: Filtered through a 37 micron Nitex mesh.

Temperature Measurements (c)

$\begin{array}{rrrrrrrr}\text { Day 0 } & \text { Day 1 } & \text { Day 2 } & \text { Day 3 } & \text { Day 4 } & \text { Day } 5 & \text { Day 6 } & \text { Day } 7 \\ 25.4 & 25.2 & 25.1 & 25.5 & 25.0 & 26.0 & 26.0 & 25.4\end{array}$


Client: Savannah River site

Sample Identification: H - F Area

Start Date: March 21, 1991

Test solutions

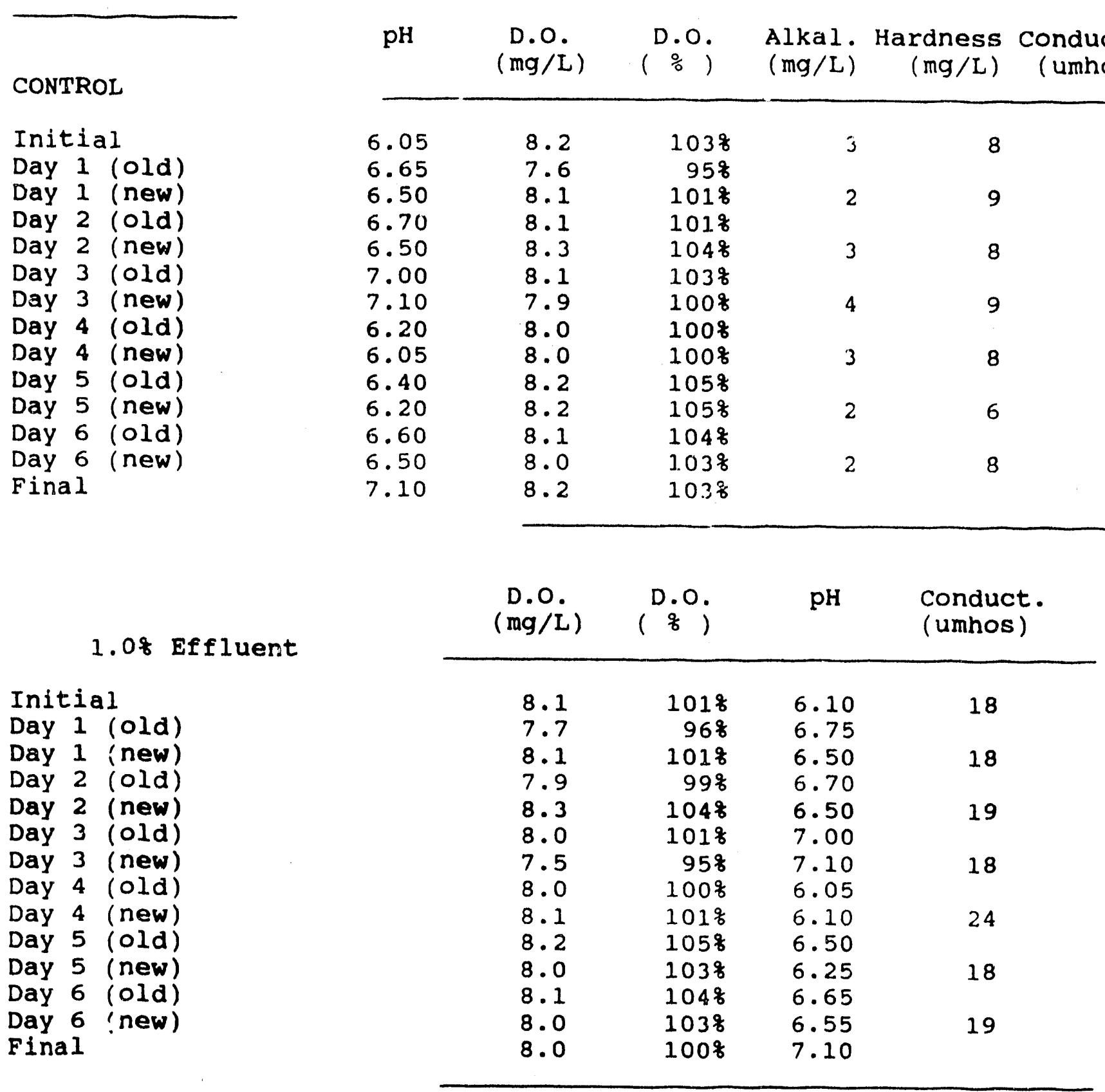


Client: Savannah River site

Sample Identification: Hi - F Area

Start Date: March 21, 1991

3.08 Effluent

Initial

Day 1 (old)

Day 1 (new)

Day 2 (old)

Day 2 (new)

Day 3 (old)

Day 3 (new)

Day 4 (old)

Day 4 (new)

Day 5 (old)

Day 5 (new)

Day 6 (old)

Day 6 (new)

Final
10.08 Effluent

Initial

Day 1 (old)

Day 1 (new)

Day 2 (old)

Day 2 (new)

Day 3 (old)

Day 3 (new)

Day 4 (old)

Day 4 (new)

Day 5 (old)

Day 5 (new)

Day 6 (old)

Day 6 (new)

Final
D. 0 .

D.O

$\mathrm{pH}$

Conduct.

(umhos )

8.0

7.7

8.0

8.0

8.1

7.9

7.6

8.1

8.2

8.2

7.9

8.2

8.1

8.0

( क )

$100 \%$

6.15

6.80

6.50

6.80

6.50

7.00

7.00

6.10

6.05

6.60

6.30

6.70

6.55

7.05

18

19

19

18

25

19

19
D. 0 .

(mg/L)
$\mathrm{pH}$

(i)
Conduct.

(umnos)

\begin{tabular}{rrrr}
8.1 & 1018 & 6.20 & 20 \\
7.8 & 988 & 6.90 & \\
8.0 & 1008 & 6.50 & 20 \\
7.9 & 998 & 6.90 & \\
8.3 & 1048 & 6.50 & 19 \\
7.9 & 1008 & 7.00 & \\
7.6 & 968 & 7.00 & 19 \\
8.0 & 1008 & 6.20 & \\
8.2 & 1038 & 6.15 & 29 \\
8.2 & 1058 & 6.65 & \\
8.1 & 1048 & 6.40 & 22 \\
8.2 & 1058 & 5.70 & \\
8.1 & 1048 & 6.75 & 22 \\
7.9 & 998 & 7.05 & \\
\hline
\end{tabular}


Client: Savannah River site

Sample Identification: $H-F$ Area

Start Date: March 21, 1991

30.08 Effluent

\begin{tabular}{|c|c|c|c|c|c|}
\hline $\begin{array}{l}\text { D. O. } \\
(\mathrm{mg} / \mathrm{L})\end{array}$ & $\left(\begin{array}{l}\mathrm{D} . \mathrm{O} \\
(q)\end{array}\right.$ & $\mathrm{pH}$ & $\begin{array}{c}\text { Alk. } \\
(\mathrm{mg} / \mathrm{L})\end{array}$ & $\begin{array}{l}\text { Hard. } \\
(\mathrm{mg} / \mathrm{L})\end{array}$ & $\begin{array}{l}\text { Cond. } \\
\text { (umhos) }\end{array}$ \\
\hline $\begin{array}{l}8.1 \\
7.9\end{array}$ & $\begin{array}{r}101 \% \\
998\end{array}$ & $\begin{array}{l}6.35 \\
6.95\end{array}$ & - & - & 22 \\
\hline $\begin{array}{l}8.1 \\
8.0\end{array}$ & $\begin{array}{l}101 \% \\
100 \%\end{array}$ & $\begin{array}{l}6.60 \\
6.90\end{array}$ & - & - & 22 \\
\hline $\begin{array}{l}8.3 \\
8.0\end{array}$ & $\begin{array}{l}1048 \\
1018\end{array}$ & $\begin{array}{l}6.60 \\
7.00\end{array}$ & - & - & 21 \\
\hline $\begin{array}{l}7.8 \\
8.0\end{array}$ & $\begin{array}{r}998 \\
100 \%\end{array}$ & $\begin{array}{l}7.00 \\
6.25\end{array}$ & $E$ & 6 & 19 \\
\hline $\begin{array}{l}3.2 \\
9.2\end{array}$ & $\begin{array}{l}103 \% \\
105 \%\end{array}$ & $\begin{array}{l}5.15 \\
6.80\end{array}$ & 5 & 6 & 37 \\
\hline $\begin{array}{l}8.3 \\
8.3\end{array}$ & $\begin{array}{l}106 \% \\
106 \%\end{array}$ & $\begin{array}{l}6.40 \\
6.70\end{array}$ & $<9$ & 6 & 30 \\
\hline $\begin{array}{l}8.2 \\
7.9\end{array}$ & $\begin{array}{r}105 \% \\
998\end{array}$ & $\begin{array}{l}6.80 \\
7.15\end{array}$ & 5 & 6 & 32 \\
\hline
\end{tabular}

Initial

Day 1 (old)

Day 1 (new)

Day 2 (old)

Day 2 (new)

Day 3 (old)

Day 3 (new)

Day 4 (old)

Day 4 (new)

Day 5 (old)

Day 5 (new)

Day 6 (old)

Day 6 (new)

Final

100.08 Effluent

pH D.O. D.O. Alkal. Hardness Cond.

$(\mathrm{mg} / \mathrm{L}) \quad(q) \quad(\mathrm{mg} / \mathrm{L}) \quad(\mathrm{mg} / \mathrm{L}) \quad$ (umhos)

Initial

Day 1 (old)

Day 1 (new)

Day 2 (old)

Day 2 (new)

Day 3 (old)

Day 3 (new)

Day 4 (old)

Day 4 (new)

Day 5 (old)

Day 5 (new)

Day 6 (old)

Day 6 (new)

Final

\begin{tabular}{|c|c|c|c|c|c|}
\hline $\begin{array}{l}7.00 \\
7.25 \\
7.20 \\
7.40 \\
7.30 \\
7.10\end{array}$ & $\begin{array}{l}8.0 \\
7.7 \\
8.0 \\
8.0 \\
8.5 \\
8.2\end{array}$ & $\begin{array}{r}1008 \\
968 \\
1008 \\
1008 \\
1068 \\
1048\end{array}$ & 10 & 4 & 34 \\
\hline- & - & & & & \\
\hline 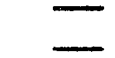 & {[} & & & & \\
\hline- & - & & & & \\
\hline- & - & & & & \\
\hline- & - & & & & \\
\hline- & - & & & & \\
\hline & & & & & \\
\hline
\end{tabular}


QDALIYY COYRELE.

\section{Client: Savannah River site}

\section{Sample Identification: H - F Area}

Start Date: March 21, 1991

\section{Culture Health:}

Sensitivity: Culture organisms were exposed to $\mathrm{NaCl}$ as a reference toxicant.

$\begin{array}{ll}\text { Current LC50: } & 0.672 \mathrm{~g} / \mathrm{L} \\ \text { Date: } 3 / 28 / 91 & \\ \text { Central Tendency: } & 0.593 \mathrm{~g} / \mathrm{L} \\ \text { Standard Dev.: } & 0.115 \mathrm{~g} \quad \\ \text { Upper Limit: } & 0.823 \mathrm{~g} / \mathrm{L} \quad(+2 \text { std. dev. }) \\ \text { Lower Limit: } & 0.363 \mathrm{~g} / \mathrm{L} \quad(-2 \text { std. dev. }) \\ \text { Current LC50 is } & 0.687 \text { standard deviation units }\end{array}$

Reproduction: Control test organisms

$\begin{array}{lrl}\text { Reproduction: } & 20.0 & \text { (young/female) } \\ \text { Central Tendency: } & 20.48 \text { (young/female) } \\ \text { Standard Dev.: } & 4.08 & \\ \text { Upper Limit: } & 28.64 \mathrm{~g} / \mathrm{L} \quad(+2 \text { std. dev.) } \\ \text { Lower Limit: } & 12.32 \mathrm{~g} / \mathrm{L} \quad(-2 \text { std. dev.) } \\ \text { Current LC50 is } & 0.118 \text { standard deviation units } \\ & & \text { below the central tendency. }\end{array}$


$+a$
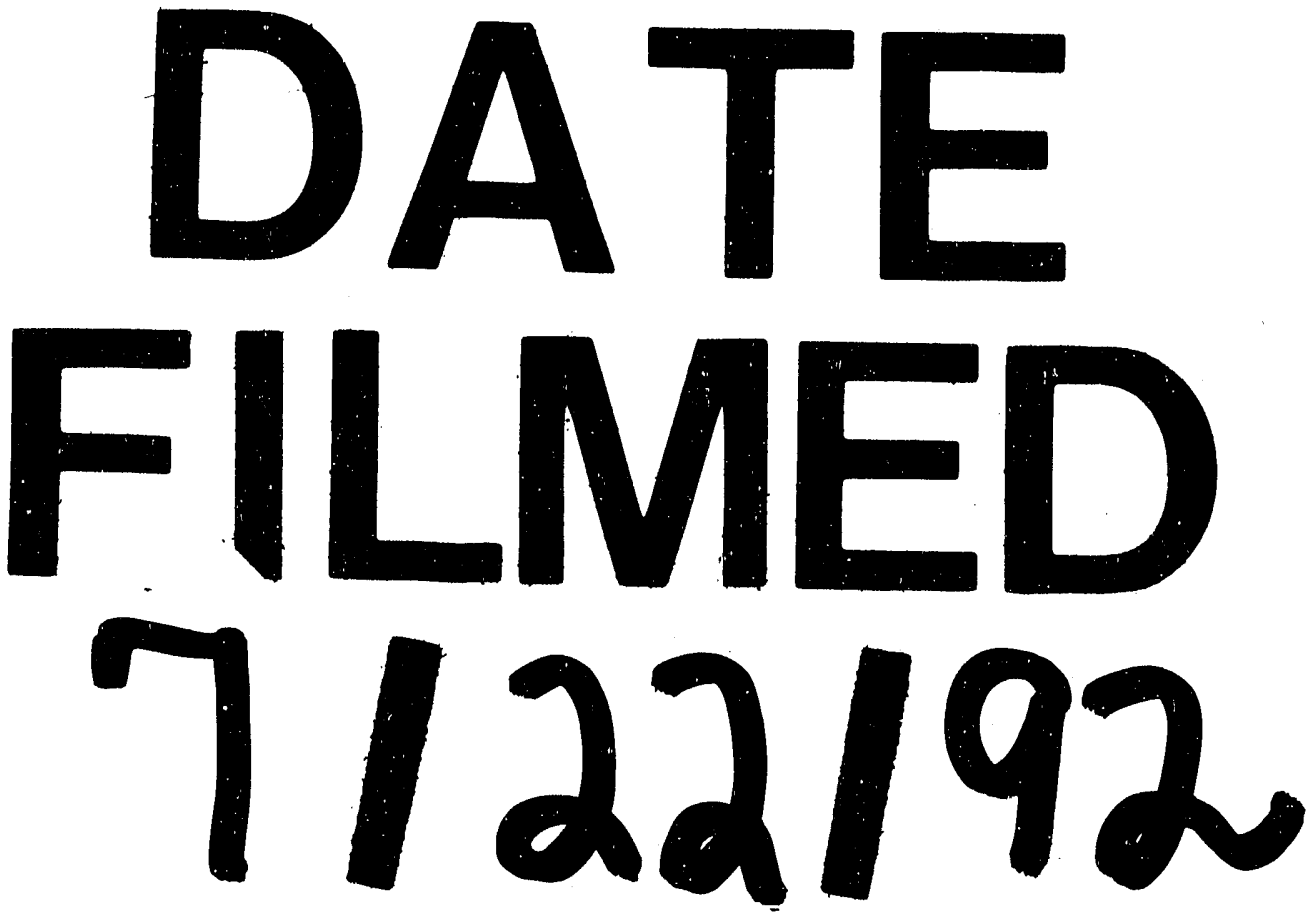
